\title{
EL DOCUMENTO SONORO Y AUDIOVISUAL DIGITAL EN EL ÁMBITO GUBERNAMENTAL... ¿DOCUMENTO DE ARCHIVO? REFLEXIONES DE UNA ARCHIVISTA
}

Debemos actuar hoy para que las generaciones futuras puedan disfrutar mañana del patrimonio audiovisual común de la bumanidad. Este patrimonio encierra enseñanzas, información y conocimientos que es esencial compartir. Es una de las bases de la identidad y la pertenencia, además de una fuente de innovación y creatividad.

Irina Bokova, directora general de la UNESCO, octubre 27, 2014

...Y lo que puede ocurrir con el tiempo es que, aunque acumulemos vastos archivos digitales, terminemos por no saber qué contienen.

Vint Cerf, uno de los padres de internet, febrero 15, 2015

\section{Resumen}

La gestión y preservación, sin un fin previsto, de los documentos sonoros y audiovisuales digitales (DSAD), presentan retos particulares para las instituciones gubernamentales o académicas y de investigación. Con frecuencia el documento sonoro (o audiovisual digital) no es considerado como un documento de archivo, por tanto, su preservación -por el tiempo que sea necesario- se ha complicado. En este contexto, se abordan la problemática, ciertos hallazgos y consideraciones sobre la preservación de los documentos audiovisuales digitales; se revisa la legislación que sienta las bases para considerar a los documentos audiovisuales y sonoros en formato digital como documentos de archivo, así como las normas actuales; finalmente, se reflexiona sobre los factores a considerar para su mejor tratamiento desde una perspectiva archivística.

Palabras clave: documentos sonoros y audiovisuales digitales, preservación, documentos de archivo, archivística.

\footnotetext{
* Consultora independiente de archivos.
} 


\begin{abstract}
The management and preservation of digital audiovisual records have specific challenges for government, academic and research institutions. The audiovisual document has not always been considered as record with serious consequences for its preservation for as long as it is needed. In this context, difficulties and some findings on preservation of digital audiovisual documents are presented, as well as certain facts are taken into consideration. Also, the legislation provides that sustain basis for considering the audiovisual document digital as a record and the regulations for these documents are briefly reviewed; finally, reflects on factors to take into account for better treatment, from archival perspective, are being considered.
\end{abstract}

Keywords: digital audiovisual records, preservation, records, records management, archival practice.

\title{
La problemática, algunos hallazgos y ciertos riesgos
}

A finales del siglo xx los documentos sonoros y audiovisuales en formatos análogos se encontraban en riesgo para su preservación, principalmente por la ausencia de equipo para su reproducción y debido a su deterioro físico; se calcula que cada década se perderá 30\% del patrimonio sonoro en Europa y $50 \%$ en América Latina. ${ }^{1}$ Parte de este patrimonio ha sido digitalizado como solución al deterioro físico; sin embargo, este proceso ha creado un nuevo problema, la preservación del contenido audiovisual digital por periodos indefinidos.

Los DSAD se extienden ampliamente; asimismo, los dueños de los archivos son heterogéneos en naturaleza; por tanto, estos materiales se encuentran en oficinas de gobierno durante su etapa administrativa, en estaciones de radio y televisión gubernamentales, o en acervos específicos destinados a su preservación; también los hay en instituciones académicas y centros de investigación, posiblemente descritos y organizados de acuerdo con ciertos estándares y preservados conforme a los esquemas de seguridad

1 Rodríguez Reséndiz, Perla, “Desafíos de la preservación digital...”, pp. 186-200. 
informática, no obstante, carecen de políticas y procesos que coadyuven a su preservación. Como consecuencia de sus cualidades, se presentan problemas específicos:

- La compresión. Por lo general los DSAD se comprimen para ser conservados; no obstante, mediante este proceso se pierde la "calidad de original", la cual no es posible recuperar en el futuro.

- La complejidad. Los archivos son tan complejos que el concepto de wrapper (envoltura) ${ }^{2}$ se desarrolló para reconocer la complejidad de los DSAD típicos, ya que contienen múltiples señales y tipos de metadatos independientes del tiempo, en ocasiones desconocidos en el ámbito de la preservación digital.

- La preservación digital. Ésta involucra la pérdida de codificaciones (vigencia de los códecs; pérdida de calidad por compresión), múltiples proxies, ${ }^{3}$ la capacidad del reproductor multimedia para decodificar la información; por ejemplo, requeriría de determinadas versiones de Windows Media, Quicktime, Flash, entre otros; pérdida de calidad como causa de edición y recombinación, así como una gama de información sobre derechos: cuestiones legales y monetarias, derechos de reproducción, preservación de tecnología de un país a otro. De igual manera, la información de estos materiales es compleja en cuanto a representar la señal, metadatos y derechos que deben preservarse. ${ }^{4}$

En el ámbito de las artes en ambiente digital, investigadores del proyecto InterPARES $2^{5}$ llevaron a cabo casos de estudio relacionados con prácticas de arte contemporáneo en diferentes categorías, tales como música, artes

2 Envoltura: estructura o programa que encapsula o contiene un grupo de datos con el propósito de facilitar su compatibilidad, uso, transmisión y almacenamiento. Voutsass y Barnard, Glosario de preservación archivística digital. Versión 4, p. 103.

$3 \mathrm{El}$ proxy es un servidor que se encuentra conectado normalmente al servidor de acceso a la Www de un proveedor de acceso y que va almacenando toda la información que los usuarios reciben de la WEB; por tanto, si otro usuario accede a través del proxy a un sitio previamente visitado, recibirá la información del servidor proxy en lugar de la del servidor real. Diccionario Babylon en: http://diccionario.babylon.com/proxy/

4 Wright, R., "Preservation of Digital Audiovisual Content. Briefing paper", p. 1.

5 Hackett, Y., Underwood, W. y Eppard, P., "Case and General Studies in the Artistic, Scientific, and Governmental Sectors...”, p. 73. 
visuales (incluían trabajos con elementos de movimiento de imágenes digitales), cine experimental y producción de documentales; en los que se encontraron diferentes visiones sobre el tratamiento de DSAD. A continuación se comentan algunas:

Actitudes y procedimientos de productores individuales o de pequeños grupos de artistas. A diferencia de la industria del entretenimiento, los artistas innovadores utilizan las tecnologías digitales, las adaptan o se inspiran por ciertas herramientas tecnológicas, sea software o hardware; es decir, exploran nuevas formas de expresión por medio de la innovación técnica; asimismo, en cuanto a producción y conservación, tanto individuos como pequeñas organizaciones adoptan recursos de punta como elemento para lograr sus intereses artísticos; sin embargo, las recomendaciones a los artistas para restringir el uso de tecnologías sólo a las que han sido ampliamente instrumentadas y sujetas a estándares internacionales, con frecuencia son percibidas como una restricción indebida a su proceso creativo y por tanto, suelen ser ignoradas.

Actitudes y procedimientos de corporaciones e instituciones de gobierno. Debido a la rápida evolución de las tecnologías, con frecuencia las organizaciones no han alcanzado la estabilización de las obras producidas en ambiente digital y abandonan u olvidan el procedimiento para enfocarse en mantener los materiales análogos que resguardan. Lo anterior es un reflejo de la impresión que se tiene de que será mejor -y más económicoreproducir los componentes digitales en plataformas digitales futuras, en lugar de migrar constantemente durante varias generaciones en ambientes que, con frecuencia, son incompatibles.

\section{Prácticas de preservación y gestión documental. Artistas o pequeños} grupos. Los estudios indican que, unos y otros, rara vez mantienen prácticas de gestión documental aceptadas: esquemas de descripción normalizados y metadatos, identificadores únicos persistentes, o documentación de acciones/transacciones respecto del uso del archivo digital o la adopción de procedimientos estandarizados. Tampoco emplean los servicios de archivistas. Por lo general, consideran que su obra es efímera, bien por falta 
de recursos o de conocimientos sobre cómo conservar los objetos de arte digital. En ocasiones intentan implementar ciertas medidas de preservación con diferentes niveles de éxito; por ejemplo, han establecido una estructura de directorio y adoptado cierta consistencia en el nombre de los objetos. El ambiente colaborativo y el libre acceso también pueden generar la producción de varias copias, esta actividad involuntaria representa la adopción del componente elemental de una estrategia de preservación conocida como LOCKss (Lot of Copies Keep Stuff Safe. Muchas copias mantienen la información segura).

Prácticas de preservación en organizaciones. Las organizaciones emplean especialistas en administración de archivos o sistemas de gestión documental y ocasionalmente acuden a los archivistas en la etapa histórica. Sus intereses económicos obligan a proteger los activos digitales producidos; por otro lado, cuentan con recursos para implementar la infraestructura y soporte necesarios para proteger sus activos digitales. El trabajo en estos ambientes involucra la contribución de expertos junto con los sistemas de gestión documental automatizados -que han sido útiles, aunque no necesarios-, para facilitar la colaboración introduciendo eficientemente los flujos de trabajo y eliminando prácticas redundantes. También proporcionan un grado de estandarización para el acceso futuro, uso y reuso, así como para proporcionar un nivel de documentación y contextualización para esfuerzos de preservación.

Los estudios indican que las organizaciones tienden a adoptar la tecnología digital cuando ha sido probada y reconocida. Básicamente, adoptan nueva tecnología con la intención de obtener acceso rápido a los materiales, así como para la obtención de beneficios financieros de acceso rápido y reuso. En estos entornos la introducción y uso de tecnología digital coexiste con prácticas de gestión documental tradicional que ya están establecidas, tales como la impresión en papel o el acceso a copias de grabaciones de audio y video análogos. 


\section{Algunas consideraciones}

Un gran volumen de DSAD se encuentra en bibliotecas y museos, videotecas o fonotecas; sin embargo, posiblemente se desconocen los aspectos relacionados con la práctica archivística útiles a la preservación -principalmente en cuanto a preservación digital- por lo que no está de más sintetizar.

La administración y la preservación de documentos de archivo digital inician a partir de la producción (incluso desde el diseño de un sistema de gestión automatizado responsable de su control), continúan durante los procesos de conservación y acceso (etapa administrativa) hasta su disposición final, definida mediante procesos de valoración documental para determinar si los documentos se dan de baja o pasan a un archivo histórico para su preservación por tiempo indefinido (etapa histórica). En principio, estos procesos no deberían excluir a los documentos de archivo por el formato o medio en el que están elaborados; es decir, pueden ser aplicados a documentos textuales, sonoros y audiovisuales o a una combinación; sólo deben considerarse los requisitos acordes con el medio, formato o soporte (en el caso de documentos de archivo análogos).

Los procesos mencionados deberían estar sustentados en un plan de archivos donde se estipulen políticas y donde los procedimientos estén basados en las diferentes normas, modelos, requisitos o estándares nacionales o internacionales que ya existen, aunque pocos hayan sido probados para los DSAD. ${ }^{6}$

La instrumentación del plan de archivos que contemple un sistema de gestión documental o administrador de contenidos automatizado garantiza la autenticidad, fiabilidad y accesibilidad de los documentos de archivo durante la etapa administrativa y, en su caso, permite mantenerla cuando se transfieren a un repositorio digital o sistema para su preservación por tiempo indefinido.

Durante la etapa de preservación es necesario contar con un plan que, entre otros, incluya los requisitos indispensables para un repositorio

6 Ibid., p. 4. 
digital: el Modelo OAIS $(4)^{7}$ resulta ser la estrategia adecuada y con mayor reconocimiento en el ámbito internacional; de igual forma, la confianza de tener un repositorio digital estará sustentada en procesos de auditoría y certificación; en consecuencia, con el propósito de dotar a las organizaciones que han adoptado el modelo OAIS con los criterios necesarios para auditarlos y certificarlos, en 2012 se emitió la ISO 16363, ${ }^{8}$ que define la práctica recomendada para evaluar la credibilidad de los repositorios digitales y garantizar que cumplan con los requisitos necesarios para preservar documentos de archivo digitales auténticos en el largo plazo. No obstante, la existencia de normas, modelos y requisitos en el ámbito internacional, los rezagos en materia de legislación y normatividad en nuestro país también representan altos riesgos.

Con respecto a los materiales digitales, es probable que las instituciones realicen esfuerzos para manejarlos, pero con carencia de políticas en la práctica cotidiana. Con frecuencia se da prioridad a aspectos tecnológicos cuando las soluciones para la producción, uso y preservación de documentos de archivo digitales está basada principalmente en procesos y controles; por tanto, si lo anterior está conduciendo a la pérdida de documentos de archivo textuales de uso común en las dependencias y entidades u organismos autónomos de gobierno, no se puede esperar menos de los DSAD. En cuanto a preservación, con frecuencia las instituciones gubernamentales sólo se centran en la información actual o corriente -aunque requiera ser utilizada por un periodo no previsible- y se olvidan de la preservación con su consecuente pérdida.

$7 \mathrm{El}$ modelo de referencia OAIS (Open Archival Information System-sistema abierto de información archivística) fue desarrollado por el Comité Consultivo para Sistemas de Datos del Espacio (CCSDS), integrado por cerca de once organizaciones gubernamentales a nivel internacional, alrededor de 28 organizaciones como observadoras y un número importante de socios de la industria. El Comité está dedicado a desarrollar normas para sistemas de información y datos del espacio. La norma ISO-14721-2012, toma como referencia el modelo.

8 La ISO 16363-2012 tiene su origen en el documento Trustwortby Respositories Audit \& certification: Criteria and checklist (auditoría y certificación para repositorios digitales de confianza. Criterios y lista de verificación, TRAC por sus siglas en inglés) fue desarrollado por los Archivos Nacionales de Estados Unidos y la CLR (Consorcio de Investigación Bibliotecaria actualmente absorbido por otro consorcio dedicado a la investigación y desarrollo de bibliotecas, la OCLC también organismo participante en su desarrollo). 
Tal vez el riesgo mayor que enfrentan los archivos digitales es la carencia de profesionales dedicados a su gestión y preservación; el panorama es el mismo para los DSAD. Es tal el volumen y descontrol que se tiene en los archivos físicos y la fascinación que ejercen para su preservación -sea por su contenido o por su calidad estética-, que el estudio y práctica de archivos digitales se ve limitada y reducida.

La ausencia de profesionalización en materia de archivos digitales es preocupante. Aunque se habla de dichos archivos, se percibe falta de formación e interés por los temas relacionados con el ambiente digital y la archivística. Con frecuencia, los materiales digitales tan sólo se abordan desde la perspectiva de las tecnologías de la información, es decir, se deja de lado la práctica archivística; no obstante, la problemática, los riesgos y otros factores como los mencionados han dado lugar a la creación de iniciativas importantes que buscan soluciones para la preservación de todo tipo de contenido digital, y en particular de los DSAD.?

En la actualidad las instituciones gubernamentales, académicas y de investigación cuentan con DSAD en proceso de producción, transmisión o difusión durante las primeras etapas de su ciclo de vida; además, cuentan con acervos que preservan archivos sonoros por tiempo indefinido; tal es el caso de la Fonoteca Nacional, institución que en su sección de preservación evidencia la problemática que enfrentan en cuanto a la identificación de los materiales y a la falta de información sobre la producción digital que conservan; ${ }^{10}$ la Cineteca Nacional cuenta con un laboratorio de restauración digital, ${ }^{11}$ donde también prevalece la falta de datos de la producción original. $^{12}$ Lo anterior, está asociado con la falta de documentos de archivo

9 Dentro de estas iniciativas se encuentran: PrestoCentre (https://www.prestocentre.org); organización sin fines de lucro dedicada a mantener vivos los contenidos audiovisuales; integra a los mejores expertos en innovación tecnológica, en audiovisuales, archivos, investigadores de medios y de toma de decisiones en diferentes foros; DAVID. Digital AV Media Damage Prevention and Repair (http:/ / david-preservation.eu), consorcio de instituciones académicas y de investigación en Europa cuyas metas son: entender, prevenir, detectar y reparar el daño de DSAD; también en el sitio https://www.prestocentre.org/4u/publication-services se localizan varios artículos y publicaciones útiles para la preservación de DSAD.

10Véase http:/ / www.fonotecanacional.gob.mx/index.php/fonoteca-nacional/preservaciony-conservacion

11 Véase http:/ / www.cinetecanacional.net/controlador.php?opcion=laboratoriodigital

12 Comentario personal de la doctora Perla Olivia Rodríguez Resendiz (Instituto de Investigaciones Bibliotecológicas y de la Información). 
que registren, desde su inicio y hasta su conclusión, toda actividad de carácter administrativo asociada a la producción, transmisión o difusión de los DSAD.

Los DSAD abundan en diferentes organizaciones de gobierno, las cuales, además de los materiales de carácter artístico cultural, producen de manera cotidiana materiales de audio y video para la promoción de acciones relacionadas con educación, salud, nutrición, agricultura, etc., cuyas probabilidades de que ya no puedan reproducirse son altas, sea porque los formatos analógicos ya no pueden leerse por la obsolescencia del equipo, porque los formatos digitales no fueron migrados en su momento y el software o hardware ya no permiten su reproducción, o porque los costos para ello son elevados.

Hasta ahora, no se tiene un cálculo del volumen de los DSAD en instituciones de gobierno o académicas. Tampoco se conocen los controles -por sus valores históricos- para su preservación. Frecuentemente, las áreas de comunicación social son las encargadas de su manejo, a pesar de que la preservación no es una función intrínseca a éstas, aunque sí lo es el manejo de los archivos producidos; pero, es probable que no se considere que los DSAD también deben ser regulados como documentos de archivo. Conforme lo anterior, la posibilidad de que gran parte de estos materiales no sean rescatables es alta.

\section{Legislación y normatividad}

Es común que los DSAD no se consideren documentos de archivo; de hecho su definición se caracteriza por el formato o el carácter cultural, mas no por las razones, funciones o procesos a los que obedece su elaboración, que pueden estar sujetos a disposiciones jurídico-normativas en el ámbito gubernamental.

En este sentido, conviene señalar que la legislación archivística en el ámbito federal tiene un alcance más allá de los textos; regularmente se piensa en los archivos digitales como semejantes a sus análogos en papel; sin embargo, cuando se revisan ciertas disposiciones jurídicas en el ámbito de acceso a la información y la legislación archivística, se tiene que: 
La Ley Federal de Trasparencia y Acceso a la Información Pública Gubernamental define, en el artículo $3:^{13}$

III. Documentos: Los expedientes, reportes, estudios, actas, resoluciones, oficios, correspondencia, acuerdos, directivas, directrices, circulares, contratos, convenios, instructivos, notas, memorandos, estadísticas o bien, cualquier otro registro que documente el ejercicio de las facultades o la actividad de los sujetos obligados y sus servidores públicos, sin importar su fuente o fecha de elaboración. Los documentos podrán estar en cualquier medio, sea escrito, impreso, sonoro, visual, electrónico, informático u holográfico;

V. Información: La contenida en los documentos que los sujetos obligados generen, obtengan, adquieran, transformen o conserven por cualquier título;

Por otra parte la Ley Federal de Archivos (LFA) ${ }^{14}$ en su artículo 4 define al documento de archivo como "el que registra un acto administrativo, jurídico, fiscal o contable, creado, recibido, manejado y usado en el ejercicio de las facultades y actividades de los sujetos obligados, independientemente del soporte en el que se encuentren".

Conforme a lo anterior, en un documento de archivo se registra un acto administrativo, jurídico, fiscal o contable, el cual se produce, maneja y usa en el ejercicio de las facultades y actividades de los sujetos obligados, con independencia al soporte en el que se encuentren; esto llevaría a diagnosticar que en la elaboración de materiales de audio y video en el ámbito gubernamental o en instituciones receptoras de presupuesto federal se debería contar con documentación a partir del inicio del proceso de elaboración o contratación y, en teoría, la primera copia original o máster también debería formar parte del documento de archivo, como derivado de la actividad regulada para desarrollar audiovisuales, todo integrado en un sistema de gestión documental automatizado (SGDA) de la institución, tal y como lo señala la LFA en su artículo $20 .{ }^{15}$ Con independencia de las copias que se elaboren para su difusión en diferentes medios. La documentación

13 Diario Oficial de la Federación, junio 11, 2002.

14 Diario Oficial de la Federación, enero 23, 2012.

15 Artículo 20. Los sujetos obligados deberán instrumentar sistemas automatizados para la gestión documental que permitan registrar y controlar los procesos señalados en el artículo 18 de la Ley para documentos de archivos, así como la elaboración, captura, organización 
y el máster del material pasarían a formar parte de los archivos de la organización.

En la actualidad, es probable que los documentos de texto relacionados con la elaboración de un audiovisual se encuentren en un SGDA en formato digital o en un expediente en formato físico; también es probable que el audiovisual sea integrado a otra base de datos o sistema -incluso, en un repositorio diferente para su conservación, uso y posible preservación-, lo que provocará que el expediente donde se documenta el proceso de elaboración se encuentre separado del producto y que su tratamiento sea diferente.

Por otra parte, tal vez, en los entornos donde se elaboran los DSAD aún no se perciben los valores informativos, probatorios o testimoniales desde la perspectiva de la rendición de cuentas, la transparencia y el derecho de acceso a la información, así como en los casos donde este tipo de materiales resultan ser evidencia para acciones de la organización o ante la justicia, durante su vida administrativa y, más adelante, en su etapa histórica.

Los valores informativos, probatorios o testimoniales van más allá de las conjeturas para el caso de los DSAD; por ejemplo, para una solicitud de acceso a información gubernamental en medio sonoro, se desconoce cuáles son las características o requisitos que garantizan que el disco o grabación que se entrega al solicitante es auténtico y fiable. También, sería importante conocer si existe alguna normativa para validar la autenticidad y que no han sido alterados los videos de vigilancia en las calles de la ciudad de México para servir, de ser el caso, como prueba auténtica y fiable ante el juez.

De igual manera, para los valores testimoniales, convendría conocer si la normativa archivística aplica a audiovisuales o películas que se producen en instituciones como, por ejemplo, el Centro de Producción de Programas Operativos y Especiales, que por su contenido deberían ser transferidos para su preservación, por tiempo indefinido, dado su valor histórico.

En cuanto a los DSAD que ya se encuentran en centros o instituciones para su preservación sin un fin previsible, estarían sujetos a los lineamientos que para ello determine el Archivo General de la Nación, como se establece en el

\footnotetext{
y conservación de los documentos de archivo electrónico procedentes de los diferentes sistemas del sujeto obligado. Esta funcionalidad deberá contar, además, con los instrumentos de control y consulta señalados en el artículo 19 de la Ley. Ley Federal de Archivos. DOF 23 de enero de 2012, disponible en: http://www.diputados.gob.mx/LeyesBiblio/pdf/LFA.pdf
} 
segundo párrafo del ya citado artículo 20 de la LFA. ${ }^{16}$ No es de sorprender que éstos sean adaptaciones de las normas vigentes en el entorno internacional.

Una vez fundamentada la calidad de los DSAD como documento de archivo en la legislación vigente, el Archivo General de la Nación es la instancia responsable de su regulación en coordinación con la Secretaría de la Función Pública y el Instituto Federal de Acceso a la Información y Protección de Datos, tal y como lo establece el capítulo II, "De la administración de los archivos", en los artículos 19, 20 y 21 de la LFA.

En relación con la normatividad para este tipo de materiales, varias instituciones dedicadas a la conservación y preservación de materiales elaboraron la Norma Mexicana NMX-R-053-SCFI-2013, documentos videográficos y fonográficos-lineamientos para su conservación, ${ }^{17}$ cuyo objetivo es "establecer los criterios y procesos para la conservación de acervos fonográficos y videográficos mexicanos, con la finalidad de reducir el deterioro de los soportes que los conforman, aumentar su vida útil y garantizar el acceso a la información que contienen". Dentro de las instituciones participantes en su elaboración están: Archivo General de la Nación, Fonoteca Nacional, El Colegio de México, Instituto Nacional de Bellas Artes, Instituto de investigaciones Dr. José María Luis Mora, Escuela Nacional de Música-UnAm, Radio Educación, por mencionar algunas.

La NMX-R-053-SCFI-2013 incluye una sección acerca de los requerimientos técnicos para la digitalización de audio y video analógico, cuyo propósito es prolongar el uso de materiales análogos. En el apartado "Sistemas de almacenamiento digital" se encuentra la sección "implementación”, que a su vez se divide en las siguientes secciones:

- Implementación. Se dispone la necesidad de establecer lineamientos de ordenación, descripción y clasificación de la información y

16 Segundo párrafo del artículo 20: "En la preservación de archivos electrónicos en el largo plazo, sea por necesidades del sujeto obligado o por el valor secundario de los documentos, se deberá contar con la funcionalidad de un sistema de preservación en el largo plazo, el cual deberá cumplir con las especificaciones que para ello se emitan".

17 En la normatividad mexicana se tienen dos tipos: las Normas Oficiales Mexicanas (NOM) y las Normas Mexicanas (NMX), las primeras son de uso obligatorio en su campo de acción, mientras que las segundas expresan recomendación de parámetros y procedimientos, aunque serán obligatorias si se hace referencia a las mismas en una NOM. 
atender las disposiciones de la Ley Federal de Derechos de Autor. Se sugiere adquirir tecnologías de la información para implementar el sistema de almacenamiento digital, equipar el almacén, conservar y tener disponible la información y permanencia de los documentos que garanticen la transferencia a otros soportes, el uso de formatos ligeros y comunes para el acceso, así como de sistemas de gestión para audio y video. Si no se tienen los recursos, la norma recomienda acudir a otras instituciones para el servicio de digitalización.

- Organización. Tendente a la optimización operativa que garantice seguridad y acceso a la información, renovar de forma periódica la tecnología de la información, estandarizar procedimientos, contar con un archivo de conservación en alta resolución con copia en formato comprimido de fácil acceso para uso y consulta, así como diferenciar copias de uso y conservación, estrategias para el mantenimiento de tecnologías (hardware, software), evaluar periódicamente políticas y procedimientos -como mecanismo para garantizar la seguridad e integridad de datos-, guardar como mínimo dos copias, para lo cual la mencionada norma dice que "puede usarse (sic) el modelo de referencia OAIS (Open Archival Information System)".

- Conservación de la infraestructura tecnológica. Advierte sobre las medidas necesarias para mantener estables los equipos de almacenamiento y la restricción de accesos, proteger los contenidos de los documentos y generar protocolos.

- Protocolos para la conservación digital. Se recomiendan acciones para contar con respaldo en almacenamiento remoto, la renovación o copia del documento digital sin alterar la información, estrategias destinadas a la actualización de software, técnicas de actualización y migración, estándares abiertos (para no comprometerse con sólo un proveedor de software), tener un solo formato para conservar todos los contenidos y uno diferente para su difusión, utilizar metadatos para resguardar, identificar y recuperar información, desarrollar procedimientos para encapsular software, metadatos y especificaciones para emulación como mecanismo para evitar disgregación de datos. 
Sin entrar en una revisión minuciosa, estos criterios son aún muy generales; sin embargo, proporcionan elementos importantes y acertados para la preservación de los DSAD. No obstante, conviene reflexionar sobre ciertos aspectos:

En los criterios se percibe una falta de conocimiento acerca del modelo OAIs, como un patrón de referencia para un sistema archivístico de información, que consiste en una organización de personas comprometidas con la preservación de materiales digitales sin un tiempo previsto, con el fin de brindar acceso a una comunidad designada de usuarios; el modelo, entre otros elementos, proporciona un esquema de entendimiento y le da relevancia al uso de conceptos archivísticos para la preservación y acceso en el largo plazo, así como un esquema para describir y comparar estrategias y técnicas de preservación; de hecho, el modelo no es solamente un medio para garantizar integridad y seguridad, parte de los propios criterios de la NMX-R-053-SCFI-2013 para el almacenamiento digital puesto que son elementos que considera el modelo OAIs.

Por otra parte, los criterios de la NMX-R-053-SCFI-2013 mencionan el desarrollo de métodos y procedimientos -aunque todavía se requiere reflexionar sobre ciertos procesos tecnológicos de conversión y actualización-. Asimismo, es interesante ver que la norma reconoce el alto costo de mantener un acervo con las características que señala y, ante la falta de recursos, se recomienda acudir a las instituciones idóneas que puedan prestar servicios de digitalización. Lo anterior reafirma la problemática de la preservación sin un fin definido de todo tipo de contenidos digitales.

De igual manera, la NMX-R-001-SCFI-2013, Documentos videográficoslineamientos para su catalogación, publicada en 2007, y actualizada en $2014,{ }^{18}$ tiene el objetivo de "contribuir a que la información que se incluya en los catálogos sea correcta, suficiente y sistematizada, con la finalidad de promover la identificación, consulta, difusión, intercambio, conservación y aprovechamiento de las obras que forman parte del patrimonio audiovisual del país". En cuanto a su alcance, está orientada preferentemente a los DSAD del ámbito educativo y cultural, sin que esto implique alguna restricción para ser aplicada por otras organizaciones. En su actualización participaron expertos de instituciones como la Asociación Nacional de Universidades e

18 No fue posible tener acceso a la NOM actual, por lo que los comentarios vertidos son de la versión 2007. 
Instituciones de Educación Superior, la Dirección General de Televisión Educativa, la Escuela Nacional de Televisión Universitaria (TV-UNAM) y el Consejo Nacional para la Cultura y las Artes, por mencionar algunas.

La NMX-R-001-SCFI-2013 pretende dar uniformidad a los elementos mínimos de descripción con el fin de lograr la recuperación sistemática y promover la preservación, conservación, consulta, difusión e intercambio de documentos audiovisuales. En vista de que los derechos de autor en los DSAD se extienden hasta su reproducción -o visión-, más allá de la copia, la norma incorpora regulación relacionada con estos derechos que no contraviene a la legislación.

La norma hace referencia a los elementos mínimos de descripción para los DSAD, en relación con las áreas de título e identificación con mención de responsabilidad, contenido, versiones, descripción técnica, disponibilidad y observaciones de producciones terminadas; también, establece las especificaciones mínimas de descripción de imágenes de archivo (imágenes asociadas, con o sin sonorización) que incluyen las áreas de título, identificación, mención de responsabilidad, área de contenido, área de descripción técnica, área de disponibilidad, área de observaciones.

En el ambiente digital la descripción es expresada en metadatos -que se definen como informaciones que dan contexto, contenido, estructura y acceso a los materiales digitales-. ${ }^{19}$ Los metadatos sirven para garantizar la autenticidad de los materiales digitales al paso del tiempo. Así, por ejemplo, se requiere de metadatos relacionados con la identidad (carácter de ser único) como nombres de personas, título, fechas, número de versión, ubicación; también los metadatos -y ciertos elementos de control- son útiles para mantener la integridad de los DSAD, como los metadatos para anotaciones posteriores a la producción, indicaciones de cambios técnicos de los materiales o a la aplicación, así como datos sobre actualización, el esquema de organización de los materiales y sus componentes digitales; de igual manera, se hace necesario contar con metadatos de auditoría y traza, así como con estrategias de protección para acceso no autorizado, existencia de copias de respaldo, políticas de seguridad y riesgos o herramientas de autenticidad. Por lo tanto, es conveniente revisar la norma mexicana para

19 UNE-ISO-23081-1:2008, Metadatos para la gestión de documentos. 
ver si se requieren nuevos elementos de descripción, sobre todo, en lo que corresponde a metadatos sobre tecnologías (hardware, software, periféricos, etc.).

\section{Para concluir}

El ideal de la preservación de los DSAD sería que estuvieran integrados en un sistema de gestión documental o administrador de contenidos que permita registrar la documentación que da lugar a su producción y divulgación -junto con el máster del DSAD-, hasta su disposición final, sea que se destruyan o se preserven sin un fin previsto en un repositorio digital bajo un esquema de políticas que aseguren su preservación. Sin embargo, la complejidad de los materiales audiovisuales, tanto en aspectos tecnológicos (formatos, software o hardware), como en derechos de autor, entre otros, inciden en la operatividad cotidiana del SGDA de una organización si no se establecen requisitos de formato y calidad.

Conforme a lo anterior, la solución pareciera ser simple para el material de audio y video mediante la preservación en repositorios con requisitos específicos vinculados al SGDA de la organización por medio de los metadatos mínimos que permitan el acceso y control de los mismos. Concluida su vida administrativa, los DSAD y el documento de archivo donde se ha mantenido la información relacionada con su proceso de elaboración, difusión, etc., pasarían -cuando así corresponda-a un sistema de preservación, de acuerdo con las disposiciones aplicables que se emitan para ello. No obstante, para encontrar una solución con el sustento adecuado, es preciso que expertos de diferentes áreas del conocimiento -relacionados con los DSAD- trabajen de forma colaborativa en la revisión del manejo de los materiales a lo largo de su ciclo de vida.

Por otra parte, la falta de control de estos materiales complica su manejo cuando se encuentran en acervos dedicados a su preservación. Debe insistirse que los trabajos desarrollados para los procesos de identificación y descripción, inventariado, conservación, digitalización, así como a restauración digital se verán limitados si no se llevan a cabo las acciones destinadas a contar con un sistema de preservación y repositorio digital que cumpla con los estándares internacionales o nacionales que para ello se establezcan y que, a la vez, sean certificados y auditados periódicamente. 
En este mismo sentido, también se hace necesario establecer alianzas con la industria de las tecnologías de la información para establecer las mejores estrategias sobre aplicaciones de software, intercambio de formatos y migración cuando se presentan problemas de divulgación o traslado a software sin que el hardware para sintetizadores o proyectores sea obsoleto, por mencionar algunas dificultades de carácter tecnológico.

La preservación de los DSAD demanda que los profesionales de la archivística y otras ciencias de la información, restauración o conservación, tecnologías de la información y comunicación, audio, cine y otros más -sean de instituciones gubernamentales, organizaciones privadas o sociales-, se actualicen y coadyuven en la formación de nuevos cuadros de profesionales. La multidisciplinariedad, la transdisciplinariedad y la interdisciplinariedad son formas de actuar ante el cúmulo de problemas a contender para lograr la preservación sin un fin previsto, por lo que los esfuerzos no pueden ser aislados.

Por otra parte, los altos costos que implica la preservación digital, invitan a reflexionar sobre la necesidad de llevar a cabo esfuerzos conjuntos y alianzas para lograr que se apliquen las mejores prácticas destinadas a uno o varios repositorios digitales donde se conjunten los DSAD provenientes de diferentes instituciones de gobierno o instituciones académicas. Aunado a ello, se vuelve necesaria la declaración de un repositorio legal de archivos públicos que preserven DSAD, sea en servidores locales o en la nube, pero que cumpla con los requisitos específicos de preservación que disponga el Archivo General de la Nación como entidad responsable de su emisión, para lo cual la participación y colaboración de los expertos en la producción de los DSAD con esa entidad será relevante.

En este trabajo se ha intentado ubicar la producción, conservación y preservación de los DSAD como documentos de archivo en instituciones gubernamentales o académicas que reciben presupuestos federales y, por tanto, están sujetas a la legislación de la Administración Pública Federal. Con ello también se ha pretendido que instituciones (bibliotecas, museos, fonotecas, audiotecas, etc.) donde se mantienen y preservan los DSAD conozcan las obligaciones a las que están sujetas; pero, además -y de mayor relevancia- que vean en la práctica archivística una estrategia que, coordinada con otras, sirva a la preservación de los DSAD. 


\section{Bibliografía}

Hackett, Y., Underwood, W. y Eppard, P., "Part One-Case and General Studies in the Artistic, Scientific and Governmental Sectors": Focus Task Force Report [versión electrónica], en: International Research on Permanent Authentic Records in Electronic Systems (InterPARES) 2: Experiential, Interactive and Dynamic Records, Luciana Duranti and Randy Preston (eds.), Padova, Italia: Associazione Nazionale Archivistica Italiana, 2008. Disponible en: http://www.interpares.org/ip2/display_file.cfm?doc=ip2_book_ part_1_focus_task_force.pdf

ISO-16363-2012. Space data an information transfer systems. Audit and Certification of trustworthy repositories. 2012 Disponible en: http://www.iso.org/iso/ catalogue_detail.htm?csnumber $=56510$

Ley Federal de Transparencia y Acceso a la Información Pública Gubernamental, Diario Oficial de la Federación, junio 11, 2002. Última reforma publicada el 14 de julio de 2014. Disponible en: http://www.diputados.gob.mx/ LeyesBiblio/pdf/244_140714.pdf

Ley Federal de Archivos, Diario Oficial de la Federación, enero 23, 2012. Disponible en: http://www.diputados.gob.mx/LeyesBiblio/pdf/LFA.pdf

Norma Mexicana NMX-R-053-SCFI-2013, Documentos videográficos y fonográficoslineamientos para su conservación. Secretaría de Economía, versión PDF. Declaratoria de vigencia publicada en el Diario Oficial de la Federación, julio 29, 2013. Disponible en: http://www.dof.gob.mx/nota_detalle.ph p? codigo $=5308323 \&$ fecha $=29 / 07 / 2013$

Norma Mexicana NMX-R-001-SCFI-2013, Documentos videográficos-lineamientos para su catalogación, Comité Técnico Nacional de Normalización, México, 2008. Declaratoria de vigencia de última versión, publicada en el Diario Oficial de la Federación, 28 de marzo de 2014. Disponible en: http://dof. gob.mx/nota_detalle.php?codigo $=5338710 \&$ fecha $=28 / 03 / 2014$

Reference Model for an Open Archival Information System (0AIS). Recommended Practices. Magenta Book. Ed. The Consultative Committee for Space Data Systems Washington, D.C. 2012. Disponible en: http://public.ccsds. org/publications/archive/650x0m2.pdf

Rodríguez Reséndiz, Perla Olivia, "Desafíos de la preservación digital de los archivos sonoros", en IV Conferencia Internacional Biredial-ISTEC. Acceso 
Aberto, Preservaçäo Digital, Interoperabilidade, Visibilidade e Dados Cientificos. Universidade Federal do Rio Grande do Sul, octubre 15-17, 2014, Porto Alegre, Brasil. Memoria del Congreso, pp. 186-200 http://biredial.ucr. ac.cr/index.php/Biredial-ISTEC_2014/2014/paper/viewFile/88/136

Trustworthy Repositories Audit and Certification. Criteria and Checklist. Ed. OCLC and CRL, 2007. Disponible en: http://www.crl.edu/sites/default/files/ d6/attachments/pages/trac_0.pdf

UNE-ISO-23081-1:2008, Información y documentación. Procesos de gestión de documentos. Metadatos para la gestion de documentos. Parte 1: Principios. Disponible en: http://www.aenor.es/aenor/normas/normas/ fichanorma.asp?tipo $=\mathrm{N} \&$ codigo $=\mathrm{N} 0041438 \#$. VO0UkfmG9cQ

Voutsass M., Juan y Barnard Amozorrutia, Alicia (coord.), Glosario de preservación archivística digital. Versión 4.0., UNAM-Instituto de Investigaciones Bibliotecológicas y de la Información, 2014. Libro electrónico disponible en: http://iibi.unam.mx/opLibros.html

Wright, R., "Preservation of Digital Audiovisual Content. Briefing paper", en Digital Preservation Europe. Disponible en: http://www.rinascimentodigitale.it/digitalpreservation/dpe-briefingpaper/audiovisual_v3.pdf 